\title{
Seguridad alimentaria y manejo sostenible agrícola con familias de pequeños productores
}

\section{Food security and sustainable agricultural management with small producers families}

\section{Segurança alimentar e gestão agrícola sustentável com famílias de}

\section{pequenos produtores}

\author{
Ardila Bernal Delia Viviana ${ }^{1}$, Rodríguez Rodríguez Mónica del Pilar ${ }^{2}$ y Acosta \\ Acosta Nieva Francisco Javier ${ }^{2}$ \\ ${ }^{1}$ Licenciada en Producción Agropecuaria, Universidad de los Llanos y \\ ${ }^{2} \mathrm{MSc}$, Docentes Universidad de los Llanos \\ mrodriguez@unillanos.edu.co
}

Recibido 08 de Agosto 2017, Aceptado 11 de Abril 2018

\section{RESUMEN}

Este trabajo se realizó en Restrepo, Meta, Colombia, en el cual participaron familias que habían sido desplazadas de sus fincas por el conflicto armado. Para el desarrollo del proyecto se utilizaron los componentes pedagógico y técnico orientados a la proyección social, con materiales didácticos como: guías de estudio, plegables, ayudas audiovisuales, visitas a los predios de algunos integrantes de la comunidad y prácticas de campo. El objetivo del trabajo fue poner en práctica el conocimiento relacionado con seguridad alimentaria y educación ambiental, temas en los cuales la comunidad ya se había capacitado, y les ha permitido mejorar su calidad de vida. Se aplicó una encuesta a las familias con el fin de conocer aspectos relacionados con los beneficios ofrecidos por el programa de formación en seguridad alimentaria, para este caso, se utilizó la educación comunitaria como mecanismo de actualización y capacitación, lo cual se realizó con el propósito de informar e indagar en la comunidad sobre qué tan efectivo fue el proceso de inclusión mediante la información de unidades agrícolas y seguridad alimentaria. Una vez analizada la fase de diagnóstico se estructuró una planeación con la comunidad de actividades tales como: calendario de visitas 
y asesorías técnicas, diseño de estrategias y herramientas pedagógicas, selección de las temáticas inherentes al manejo de tecnologías apropiadas para cultivos de hortalizas, frutales, maíz y caña; y planeación de actividades de extensión como demostraciones de método, días de campo y giras. Se entregaron a las familias insumos y materiales necesarios para desarrollar el proyecto de seguridad alimentaria y las actividades de asesoría técnica. De acuerdo al cronograma concertado, se realizaron las visitas de acompañamiento y de asesoría a las familias que dieron continuidad a su proyecto siguiendo un manejo sostenible de unidades agrícolas familiares. El $86 \%$ de los productores indicaron que sí habían desarrollado alguno de los proyectos de seguridad alimentaria: 29\% han establecido huertas familiares, $7 \%$ avicultura solamente, $7 \%$ avicultura y piscicultura, 7\% avicultura, huerta y piscicultura, y 43\% han implementado avicultura y huerta. Las estrategias educativas utilizadas en el proceso de proyección social fueron exposiciones, discusiones, talleres, días de campo, clases teórico prácticas y demostración de métodos; los cuales se consideraron pertinentes por parte de la comunidad, quienes manifestaron que estas actividades facilitaron el aprendizaje porque se utilizó un lenguaje sencillo.

Palabras clave: Proyecto pedagógico, agricultura familiar, estrategias educativas.

\section{ABSTRACT}

This work was carried out in Restrepo, Meta, Colombia, in which participated families who had been displaced of their farms because of the armed conflict. For the development of the project the pedagogical and technical components oriented to the social projection were used, with didactic materials such as: study guides, folding, audiovisual aids, visits to the premises of some members of the community and field practices. The objective of the work was to put into practice the knowledge related to food security and environmental education, themes in which the community had already been trained, and has allowed them to improve their quality of life. A survey was applied to families in order to know aspects related to the benefits offered by the food safety training program, for this case, community education was used as an updating and training mechanism, which was carried out 
for the purpose of informing and inquiring into the community about how effective was the inclusion process through the information of agricultural units and food security. Once the diagnostic phase was analyzed, a planning was structured with the community of activities such as: schedule of visits and technical advice, design of strategies and pedagogical tools, selection of the themes inherent to the management of appropriate technologies for vegetable, fruit, corn and sugarcane crops; and planning of extension activities such as demonstrations of method, field days and tours. The families were provided with supplies and materials necessary to develop the food security project and technical advisory activities. According to the agreed schedule, accompaniment and counseling visits were made to the families who gave continuity to their project following a sustainable management of family agricultural units. The $86 \%$ of the producers indicated that they had developed some of the food security projects: $29 \%$ have established family orchards, $7 \%$ poultry only, $7 \%$ poultry and fish farming, $7 \%$ poultry, orchard and fish farming, and $43 \%$ have implemented poultry and vegetable orchards. The educational strategies used in the process of social projection were exhibitions, discussions, workshops, field days, practical theoretical classes and demonstration of methods; which were considered relevant by part of the community, who stated that these activities facilitated learning because simple language was used.

Keywords: Pedagogical project, family farming, educational strategies.

\section{RESUMO}

Este trabalho foi realizado em Restrepo, Meta, Colômbia, em que participaram famílias que haviam sido deslocadas de suas fazendas por causa do conflito armado. Para o desenvolvimento do projeto foram utilizados os componentes pedagógicos e técnicos orientados para a projeção social, com materiais didáticos, tais como: guias de estudo, dobras, recursos audiovisuais, visitas às instalações de alguns membros da comunidade e práticas de campo. O objetivo do trabalho foi colocar em prática os conhecimentos relacionados à segurança alimentar e educação ambiental, tópicos em que a comunidade já havia sido treinada e lhes permitiu melhorar sua qualidade de vida. Uma pesquisa foi aplicada às famílias 
para conhecer aspectos relacionados aos benefícios oferecidos pelo programa de treinamento em segurança alimentar, para este caso, a educação comunitária foi usada como um mecanismo de atualização e treinamento, que foi realizado com a finalidade de informar e investigar na comunidade sobre quão eficaz foi o processo de inclusão através de informações sobre unidades agrícolas e segurança alimentar. Uma vez analisada a fase de diagnóstico, um planejamento foi estruturado com a comunidade de atividades como: agenda de visitas e assessoria técnica, desenho de estratégias e ferramentas pedagógicas, seleção dos temas inerentes à gestão de tecnologias apropriadas para as culturas de vegetais, frutas, milho e cana-de-açúcar; e planejamento de atividades de extensão, como demonstrações de método, dias de campo e passeios. As famílias receberam suprimentos e materiais necessários para desenvolver o projeto de segurança alimentar e as atividades de assessoria técnica. Seguindo com o cronograma acordado, se realizaram as visitas de acompanhamento e aconselhamento às famílias que deram continuidade ao seu projeto seguindo uma gestão sustentável das unidades agrícolas familiares. $0 \quad 86 \%$ dos produtores indicaram que haviam desenvolvido alguns dos projetos de segurança alimentar: $29 \%$ estabeleceram hortas familiares, $7 \%$ somente avicultura, $7 \%$ aves e piscicultura, 7\% aves, horta e piscicultura, e 43\% implementaram aves e hortas. As estratégias educativas utilizadas no processo de projeção social foram exposições, discussões, oficinas, dias de campo, aulas teóricas práticas e demonstração de métodos; que foram considerados relevantes por parte da comunidade, que afirmaram que essas atividades facilitavam o aprendizagem porque foi utilizado uma linguagem simples.

Palavras-chave: Projeto pedagógico, agricultura familiar, estratégias educacionais.

\section{INTRODUCCIÓN}

El concepto de seguridad alimentaria aparece en la década de los años 1970, el cual ha evolucionado desde consideraciones de tipo cuantitativo y económico hacia una definición que tiene en cuenta la dimensión humana del fenómeno. 
Años después la seguridad alimentaria se definió como la "capacidad en todo momento de aprovisionar los productos básicos", de modo que se puede sostener un crecimiento del consumo alimentario, soportando las fluctuaciones y los precios. Posteriormente la definición incluía la capacidad de asegurar que el sistema alimentario provea a toda la población una adecuada nutrición a largo plazo (PESA, 2011).

El concepto de seguridad alimentaria hace referencia a la disponibilidad suficiente y estable de alimentos, y al el acceso y consumo oportuno y permanente de los mismos en cantidad, calidad e inocuidad por parte de todas las personas, bajo condiciones que permitan su adecuada utilización biológica, para llevar una vida saludable y activa. Este enfoque pone de manifiesto que todas las personas tienen derecho a contar con una alimentación suficiente, oportuna y adecuada, y que no deben carecer de alimentos básicos en su canasta familiar (GNC, 2007; Moreno y Fidélis, 2012).

Se entiende como sistema productivo sostenible un conjunto particular de actividades desarrolladas en el medio rural para obtener ciertos bienes o servicios con la intención de comercializarlos, y que se caracteriza por ciertas formas de uso del patrimonio natural local (sistema de manejo) que no degradan progresivamente su capacidad productiva; tales actividades pueden ser propiamente productivas: cultivo, pecoreo, colecta, aprovechamiento, extracción, pastoreo o prevención, mantenimiento, y restauración (Chapin et al., 2004; Crona y Parker, 2012).

Un manejo sostenible del agroecosistema queda definido por una equilibrada combinación de tecnologías, políticas y actividades, basada en principios económicos y consideraciones ecológicas, a fin de mantener o incrementar la producción agrícola en los niveles necesarios para satisfacer las crecientes necesidades y aspiraciones de la población mundial en aumento, pero sin degradar el ambiente (Martínez, 2009). 
Se refiere al uso, desarrollo y protección de los recursos, tanto naturales como físicos, a una tasa que permite a las personas y comunidades proveerse de bienestar social, económico y cultural en beneficio de su salud y seguridad, mientras se mantiene su potencial original a lo largo del tiempo. Es un concepto dinámico que parte de un sistema de valores emanado del contexto social y ambiental imperante (MSSSI, 2013).

Dentro de este contexto, los proyectos pedagógicos productivos (PPP) son una herramienta para la transformación participativa y comunitaria a partir del diseño y puesta en marcha de proyectos agro-pedagógicos pertinentes, amigables con el medio ambiente, y sostenibles. Puede considerarse como la dedicación por parte de la comunidad educativa a la producción con el fin de acrecentar sus bienes económicos sociales y culturales. Los PPP tienen por beneficiarios a la comunidad educativa a nivel local y deben dimensionar las circunstancias particulares donde se va a realizar el proceso de proyección social, y ser pertinentes a las necesidades de la comunidad, valorando sus potencialidades culturales, artísticas y deportivas, para finalmente desarrollar sus saberes locales (Castillo, 2004).

Desde hace más de una década en el departamento del Meta, Colombia se vienen incrementando situaciones de desplazamiento forzado, por el conflicto armado, aumentando la migración de familias campesinas, frente a lo cual los gobiernos locales, han tomado medidas para tratar de mitigar la problemática de las comunidades afectadas por su vulnerabilidad, que generalmente son pequeños productores agrarios (MSPS y FAO, 2012).

En Restrepo, la Universidad de los Llanos en conjunto con ASCUN y la Alcaldía municipal, realizaron visitas a los predios de aquellas personas que dieron continuidad al proyecto de seguridad alimentaria establecido con anterioridad, donde se tienen implementadas unidades agrícolas, avícolas y piscícolas. Teniendo en cuenta lo anterior, se determinó que hubo cumplimiento de los objetivos que fueron desarrollados a través de procesos educativos que promueven la salud y la seguridad alimentaria, también se otorgaron aves, herramientas, insumos como mallas y semillas. 
Por lo anterior el objetivo del presente estudio fue continuar fortaleciendo los procesos de formación en la comunidad teniendo en cuenta el interés que mostraron por aprovechar el conocimiento apropiado en seguridad alimentaria y educación ambiental, lo cual les ha permitido mejorar su calidad de vida; los que pudieron continuar con la implementación de los proyectos pedagógicos productivos y los que actualmente no cuentan con predios lograron fortalecer el capital semilla entregado, además de mantener el interés por profundizar en estos temas. Los productores confiaron en ser incluidos verdaderamente en igualdad de condiciones, para poder poner en práctica dichos conocimientos.

Estratégicamente y para poder orientar la realización de este proyecto, se tuvo en cuenta para la fase de diagnóstico, la revisión de la información de la caracterización realizada en el año 2012 por la Alcaldía de Restrepo y la Universidad de los Llanos, siendo un insumo importante para identificar especialmente a las familias que participaron en la formación dentro del proceso de inclusión para la generación de seguridad alimentaria.

\section{ACTIVIDADES DESARROLLADAS}

Este trabajo se realizó en Restrepo, Meta, Colombia, que hace parte de los 29 municipios del Departamento del Meta, se encuentra ubicado a 4은 16 latitud norte y $73^{\circ} 34^{\prime} 25^{\prime \prime}$ de longitud oeste del Meridiano de Greenwich y a $570 \mathrm{msnm}$. La temperatura varía entre 17.2 y $34.4^{\circ} \mathrm{C}$ siendo la media de $25.8^{\circ} \mathrm{C}$. La humedad relativa es $83 \%$, alcanzando valores de $93 \%$ en la época lluviosa y $56 \%$ en la época seca (IDEAM, 2016).

Participaron 38 personas cabeza de familias, que corresponden al $100 \%$ de la población vulnerable beneficiada con el proyecto de seguridad alimentaria establecido con anterioridad. Se trabajó con 14 familias de la población vulnerable como muestra representativa, teniendo en cuenta que las demás personas han cambiado de ciudad. Para el desarrollo de este proyecto se utilizaron los componentes pedagógico y técnico, orientados a la proyección social con materiales didácticos como: guías de estudio, plegables, ayudas audiovisuales, 
visitas a los predios de algunos integrantes de la comunidad y prácticas de campo. El proceso de evaluación se realizó mediante encuesta y entrevista, lo que permitió la recolección de información para responder a los objetivos.

Se realizó una encuesta a las familias con el fin de conocer aspectos relacionados con el proceso de inclusión y los requisitos que cumplieron para poder hacer parte de los beneficios ofrecidos dentro del programa de formación en seguridad alimentaria. Para este caso, se utilizó la educación comunitaria como mecanismo de actualización y capacitación, con el fin de que los objetivos propuestos llegaran a su finalidad. Este proceso se realizó con el propósito de informar e indagar en la comunidad qué tan efectivo fue el proceso de inclusión mediante la formación de unidades agrícolas y seguridad alimentaria.

Una vez analizada la fase de diagnóstico se estructuró una planeación con la comunidad, actividades tales como calendario de visitas y asesorías técnicas; diseño de estrategias y herramientas pedagógicas; selección de las temáticas inherentes al manejo de tecnologías apropiadas en la producción de hortalizas y cultivos de pancoger; y actividades de extensión como demostraciones de método, días de campo y giras.

Se entregaron a las 14 familias insumos y materiales necesarios para desarrollar el proyecto de seguridad alimentaria y las actividades de asesoría técnica, y de acuerdo a un cronograma concertado, se realizaron las visitas de acompañamiento y de asesoría a las familias que dieron continuidad a su proyecto de seguridad alimentaria y manejo sostenible de unidades agrícolas familiares.

La supervisión y evaluación continuada de los procesos formativos y académicos de las familias, así como de los de capacitación y de adopción de tecnologías apropiadas en el manejo de la huerta, también se evaluó la utilización de los insumos agropecuarios entregados a cada familia, obtenidos con recursos de la Alcaldía de Restrepo.

Para facilitar el trabajo con los pequeños productores las estrategias utilizadas para el proceso de aprendizaje fueron: demostración de métodos, días de campo, 
exposiciones, clases teórico-prácticas y grupos focales, las familias expresaron que estas metodologías fueron pertinentes, porque se utilizó un lenguaje elemental, de fácil comprensión, y resaltan que las prácticas y visitas a los predios de las familias fueron oportunidades que permitieron interactuar con los estudiantes haciendo más comprensible los conocimientos (Figura 1).
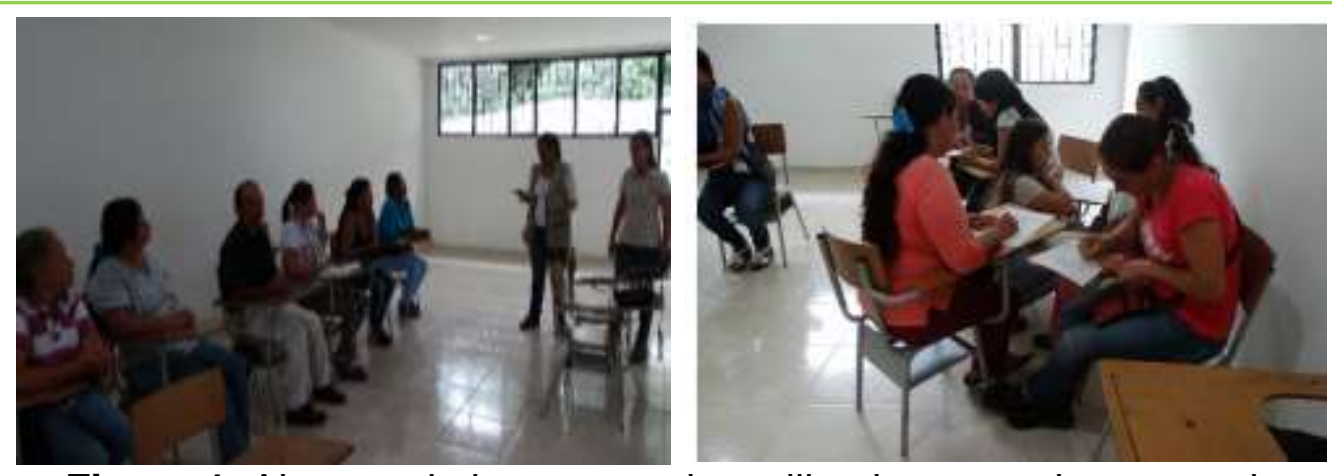

Figura 1. Algunas de las estrategias utilizadas para el proceso de aprendizaje fueron: demostración de métodos, exposiciones, clases teóricoprácticas

La entrevista a los miembros de la comunidad, permitió conocer su opinión frente a lo que ha realizado las instituciones gubernamentales con ellos, así mismo, saber si estarían interesados en continuar siendo participes de formación enfocada a mejorar su calidad de vida; de igual forma, ser testigos si los conocimientos adquiridos y proyectos productivos aportados por parte de la Universidad han influenciado en su bienestar.

\section{RESULTADOS}

Las familias manifestaron que el trabajo realizado por parte de la Universidad ha sido positivo y excelente; así mismo, indicaron estar interesados en continuar siendo participes en procesos de formación o proyectos productivos. Durante la ejecución del proyecto se realizaron actividades como: socializaciones, debates y grupos focales, en las cuales la comunidad dio a conocer su punto de vista respecto a las temáticas abordadas en seguridad alimentaria, atendiendo correctamente a cada una de las observaciones y sugerencias; lo anterior se 
realizó con el fin de identificar sí las estrategias utilizadas en el proceso de formación fueron las adecuadas para generar cambios en ellos.

Como estrategia de motivación, se hizo necesario ofrecer a la comunidad talleres de actualización en seguridad alimentaria; este proceso se cumplió teniendo en cuenta la información recolectada en la encuesta donde se consultó a la comunidad los temas de interés, a lo cual el $86 \%$ respondieron que estaban dispuestos a recibir capacitación en temas en referentes a la seguridad alimentaria como: piscicultura, cunicultura, avicultura y huerta familiar; además se realizó actualización en inyectología y heliconias (Figuras 2 y 3).

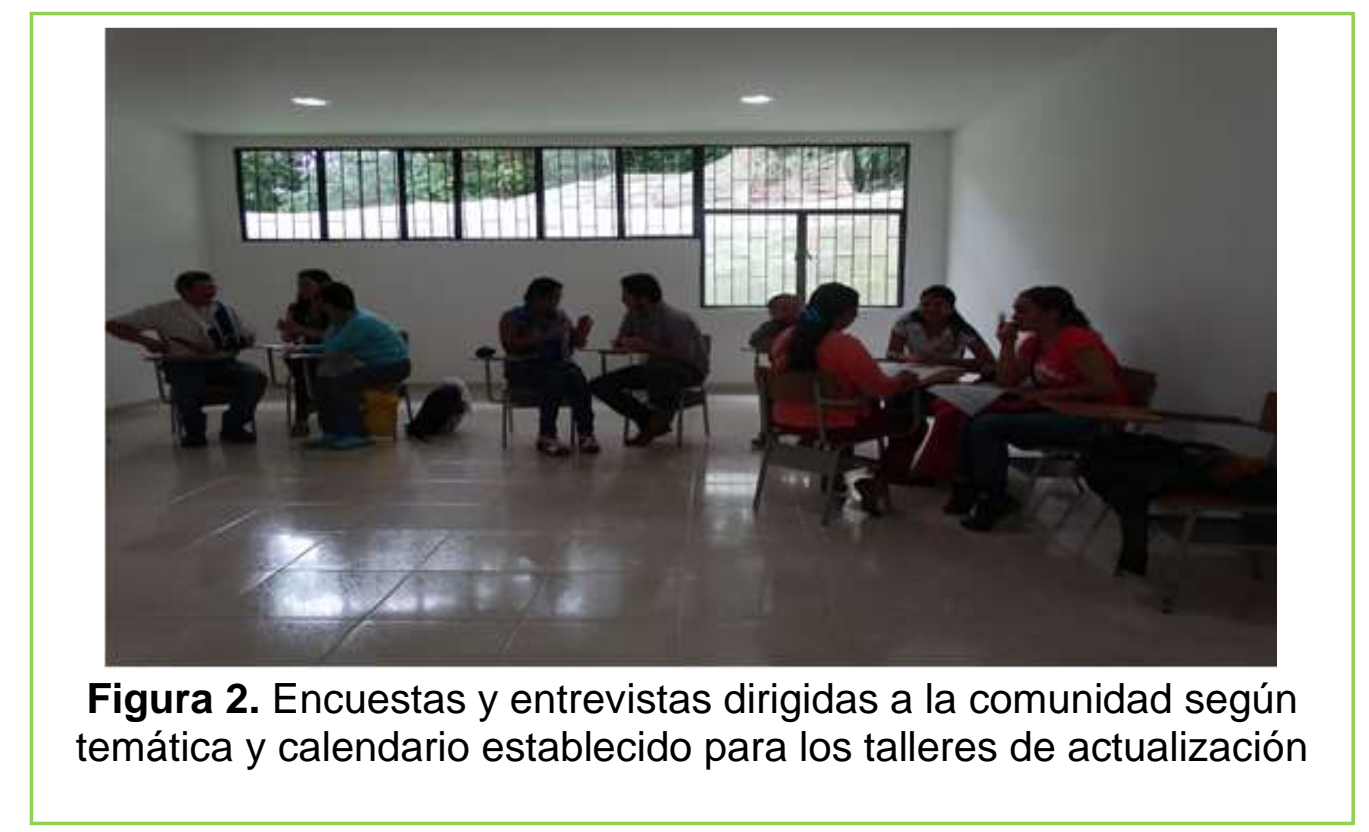

Con el fin de determinar cuáles fueron los cambios generados en la comunidad a partir del proceso de formación, se encontró que el 100\% respondió que obtuvo conocimientos. Referente a un proceso de integración social el $71 \%$ de las personas manifestaron que se dio dicho proceso, y el $28 \%$ afirmaron que hubo acercamiento con sus compañeros de formación; en cuanto a si han obtenido ingresos económicos a partir de estos proyectos, el $28 \%$ indicaron que sí se ha beneficiado, mientras que el $57 \%$ informaron que han sido muy pocas las entradas que se han generado, por último el $14 \%$ afirmaron que no han logrado ningún 
ingreso. Respecto a una mejora en su calidad de vida el $86 \%$ expresaron que han incrementado su calidad de vida y el $14 \%$ manifestaron que ha aumentado muy poco en este aspecto (Gráfica 1).
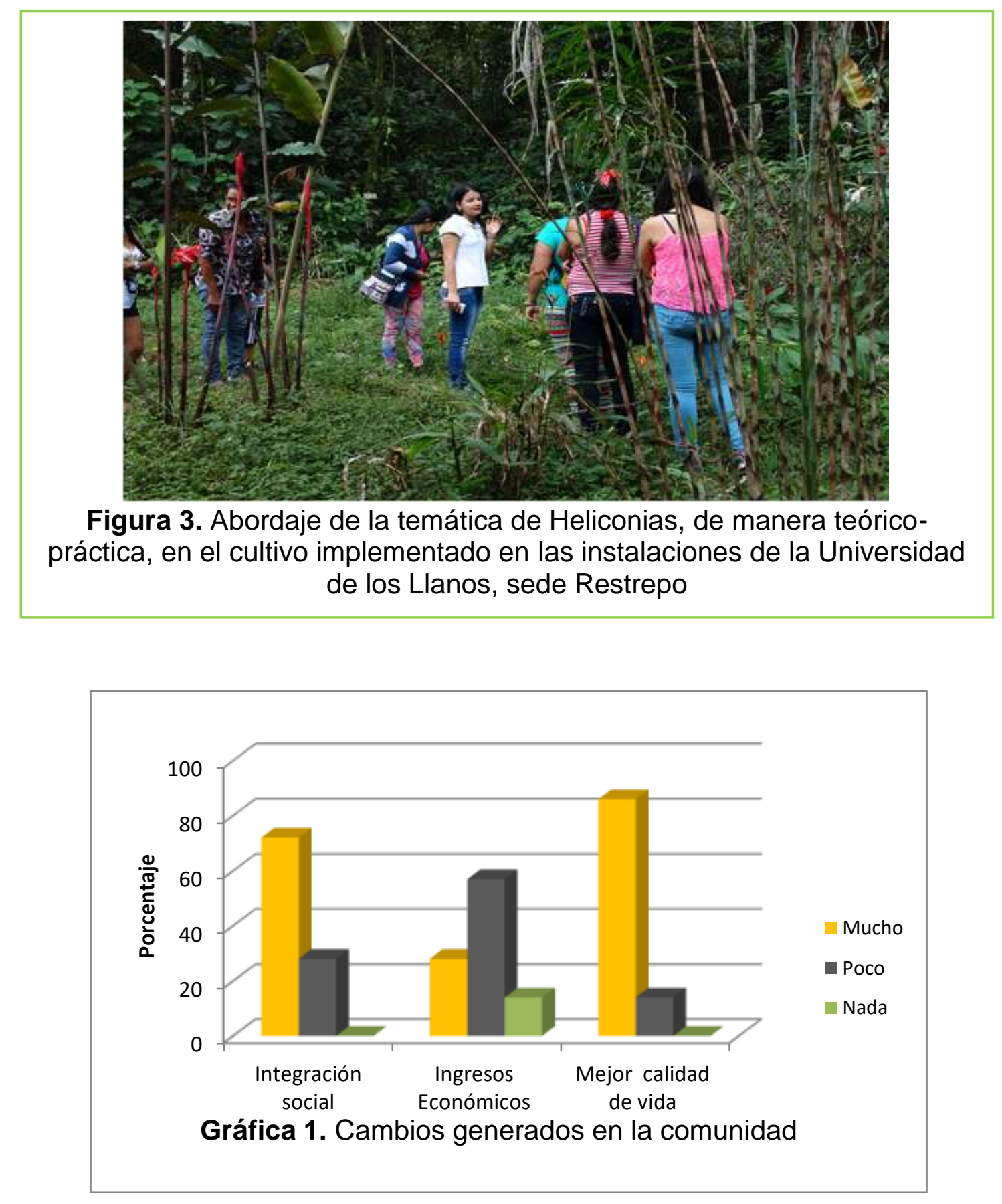

El $86 \%$ de los productores indicaron que sí han implementado alguno de los proyectos de seguridad alimentaria, mientras que el $14 \%$ respondió que no lo han 
hecho. Al estipular cuales han sido estos proyectos mencionaron que un $29 \%$ han establecidos huertas familiares, $7 \%$ avicultura, $7 \%$ avicultura, huerta y piscicultura, $7 \%$ avicultura y piscicultura, y $43 \%$ han implementado avicultura y huerta (Gráfica 2).

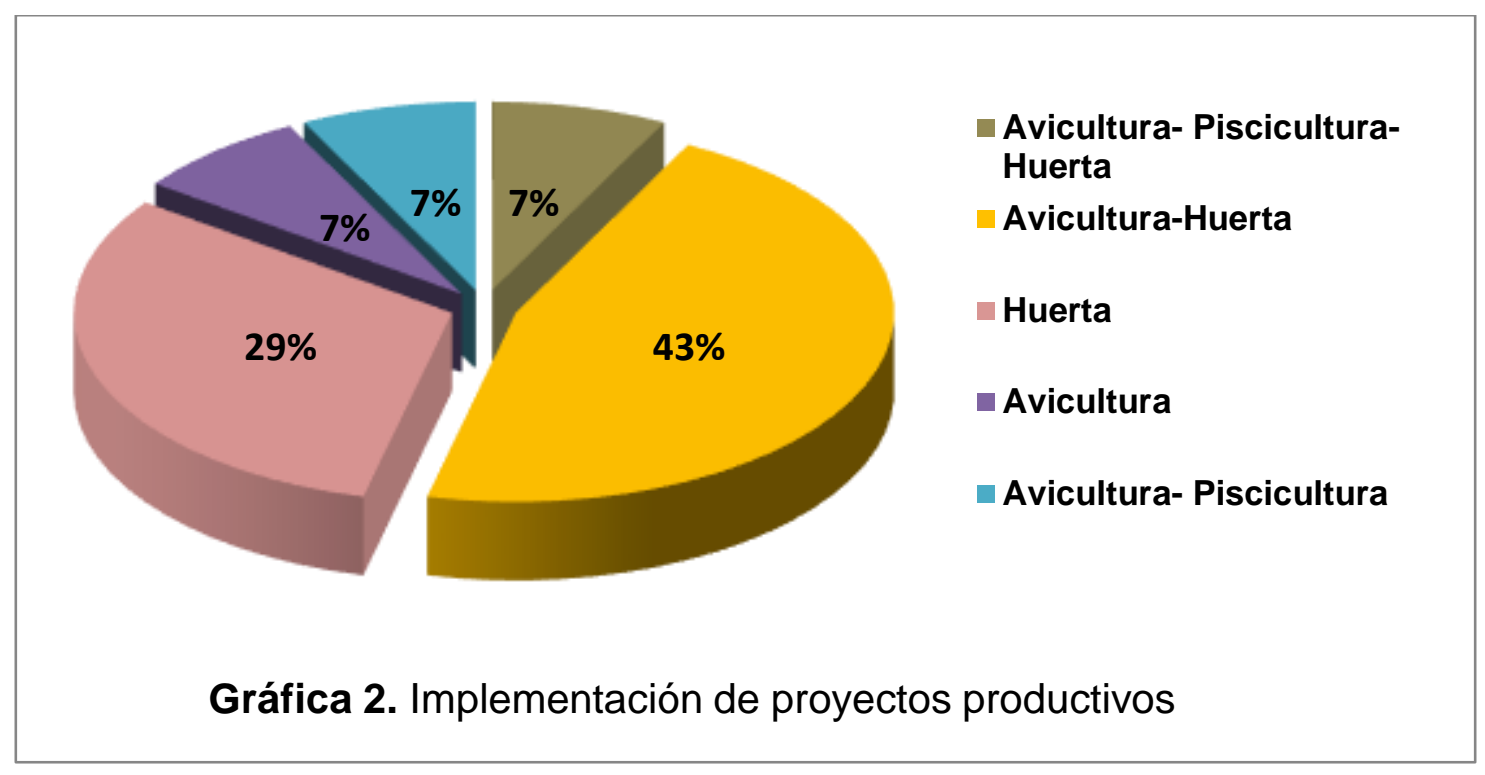

\section{ANÁLISIS DE ACTIVIDADES CON LA COMUNIDAD}

De acuerdo con la ley de víctimas y el programa de apoyo al desplazado, planteado por el actual gobierno nacional, se encuentra un proceso de inclusión centrado principalmente en el derecho a la igualdad, lo cual involucra aspectos relacionados con: vivienda, educación, familia, empleo, vestido y salud, se destaca que el índice de inclusión social no queda relegado únicamente a la pobreza, sino a indicadores como la educación, la salud, la economía y la reconstrucción social.

Teniendo en cuenta lo anterior para el caso específico de las familias que participaron en estos proyectos, el proceso de inclusión se evaluó respondiendo a las siguientes variables: aportes a la calidad de vida, trasferencia y aplicación del conocimiento y construcción social del proceso de formación en seguridad alimentaria y educación ambiental. Este proceso de evaluación se abordó inicialmente con una presentación ante la comunidad, donde se dieron a conocer los propósitos y objetivos del proyecto, seguidamente se aplicó una encuesta y una entrevista que ayudó a recolectar información esencial para dar cumplimiento 
a los objetivos propuestos; entre la información recogida se encontró qué la comunidad debía cumplir con ciertos requisitos para ser parte del proceso de inclusión, a lo que ellos respondieron que eran necesarios sus datos personales para confrontar la información con la base de datos de personas en situación de desplazamiento que reposa en la Alcaldía de Restrepo; de igual manera las familias debían demostrar el acceso a un predio que permitiera implementar los proyectos productivos y ser personas en situación de vulnerabilidad. Al mismo tiempo, expresaron mediante la encuesta que el trabajo realizado por parte de la Universidad ha sido positivo, puesto que estos procesos han sido oportunidades de mejorar sus condiciones de vida, porque en un primer momento no solo accedieron al conocimiento, sino que implementaron los proyectos en sus viviendas para obtener recursos y alimentación, gozando así de un bienestar socioeconómico.

Simultáneamente la comunidad manifestó interés en continuar procesos de formación $y / o$ proyectos productivos, puesto que esto ayuda a brindar oportunidades de mejorar sus condiciones de vida, a generar interés por seguir buscando alternativas, y continuar perfeccionando sus conocimientos, teniendo en cuenta los beneficios de la seguridad alimentaria al permitirles proveer su propio sustento, consumo o la posibilidad de generar ingresos. Se realizaron actividades como socializaciones y debates, las cuales incidieron en la identificación de las estrategias utilizadas en el proceso de formación para así determinar sí estas fueron las apropiadas para lograr cambios generados en la comunidad. En relación con lo anterior, la comunidad expresó que las estrategias de demostración de métodos, días de campo, exposiciones, clases teórico-prácticas y grupos focales, les permitió adquirir y aplicar los conocimientos en sus predios mejorando sus condiciones de vida. También se realizaron talleres de actualización en temas de seguridad alimentaria como piscicultura, cunicultura, avicultura y huerta familiar; además se realizó una actualización en inyectología y heliconias, la cual se llevó a cabo con el apoyo de los estudiantes del curso de educación y extensión comunitaria, quienes utilizaron como estrategias educativas la demostración de métodos, días de campo, exposiciones y clases teórico-prácticas, 
y herramientas como ayudas audiovisuales, maquetas, folletos y carteleras, siendo estas necesarias para que la comunidad fuera participe y alcanzara de la mejor manera estos procesos de refuerzo formativo.

El tema de heliconias fue dirigido a la comunidad mediante un día de campo, se les mostró y explicó las clases de heliconias, características, tipo de suelo y temperatura, labores culturales y propagación, ellos se mostraron interesados al respecto, por ser una especie ornamental promisoria.

Dando continuidad a las actividades se realizaron clases teórico prácticas según el calendario acordado, el tema que se abordó fue cunicultura, donde la comunidad tuvo la oportunidad de interactuar con los estudiantes acerca del manejo de la producción del conejo en aspectos como alimentación, sexaje, instalaciones, razas, gestación y reproducción, así mismo se escucharon sus conocimientos empíricos o procesos que realizaban en sus predios, siendo un espacio interesante tanto para los estudiantes como para la comunidad. Dentro de esta actividad se realizó demostración de método en los temas de piscicultura y avicultura, donde se le enseñó el sistema de jaula flotante, para que de esta manera ellos puedan llevarlo a práctica, además de mostrar cómo era el proceso de trasplante de los alevinos al pozo; en cuanto a la temática de avicultura se dio a conocer el manejo de infraestructura, agua, comida y sexaje, en esta misma instancia se dio un refuerzo en el tema de huerta familiar en temas como: métodos de siembra, labores culturales, tipos de semilleros caseros, riegos y drenajes, hortalizas y cultivos de pan coger. Para dar final a la agenda de las actividades y horario pactado se entregó a la comunidad semillas de hortalizas, peces y aves de corral que fueron utilizados en la demostración de métodos.

Se recolectó información que permitió demostrar los aportes y cambios generados en la comunidad, se experimentó un proceso de unificación social entre los participantes del proyecto, puesto que habían espacios de interacción, discusión e integración, respecto a los ingresos económicos la mayoría informó que no les alcanzó el nivel de producción para comercializar, sino que solo fue para su consumo, y finalmente respecto a una mejora en su calidad de vida el $86 \%$ 
expresó que a partir de estos proyectos se ha incrementado debido a que han adquirido alimento, conocimiento, ingresos, unión familiar y social, y el $14 \%$ manifestó que ha aumentado muy poco su calidad de vida porque sus necesidades son extensas y aunque consiguen oportunidades con estos proyectos, no le son suficientes para suplirlas.

Partiendo de los supuestos anteriores se consultó a la comunidad si ellos habían implementado proyectos productivos en sus predios, a lo cual el $86 \%$ respondió que afirmativamente, entre los que se destacan: avicultura, piscicultura y huerta familiar; usando así los conocimientos adquiridos y dándoles como resultado alimentación, sustento e ingresos. Estas personas que implementaron proyectos productivos lo hicieron porque contaban con el lugar, tiempo y recursos necesarios para la actividad; mientras que el $14 \%$ reveló que no lo hicieron debido a que los predios en los que residían eran de arrendatarios o tenedores.

El proceso de inclusión se centró en la aplicación de estrategias educativas que permiten la comprensión de las temáticas abordadas, también se desarrollaron elementos de construcción social, puesto que se convirtió en el centro de reunión y retroalimentación de experiencias, vivencias e historias de vida que terminaron fortaleciendo de esta manera lazos de amistad; al estar vinculados en las actividades cotidianas de la comunidad junto a los encargados del proceso, permitió una relación más estrecha basada en la animación permanente a relacionarse en actividades, donde había espacio de interactuar mediante procesos de días de campo, talleres de formación y visitas a sus predios. Con base a esto surgió el proceso de evaluación de estas estrategias, donde se reforzaron temáticas que fueron acordadas por parte de la misma comunidad.

\section{CONCLUSIONES}

Las estrategias educativas utilizadas en el proceso de proyección social fueron exposiciones, discusiones, talleres, días de campo, clases teórico-prácticas y demostración de métodos, que se determinaron como pertinentes por parte de la 
comunidad quienes manifestaron que estas actividades facilitaron la comprensión del aprendizaje porque se utilizó un lenguaje sencillo.

Se realizaron talleres de actualización en huerta familiar, heliconias, cunicultura, piscicultura e inyectología, complementariamente al proceso de formación en seguridad alimentaria y unidades agrícolas familiares, mostrándose un alto interés por parte de la comunidad para implementarlos.

Los factores de selección que incidieron en el proceso de inclusión permitieron generar cambios importantes en la comunidad tales como: conocimientos y construcción social, dejando así, un legado significativo en la formación, integración y actitud frente al deseo por mejorar su calidad de vida.

\section{RECOMENDACIONES}

El acompañamiento constante a la comunidad rural es importante en un proceso formativo, puesto que se genera compromiso y responsabilidad durante las diferentes fases que componen la proyección social. Teniendo en cuenta lo anterior, se propone que la Universidad genere procesos de formación e investigación en el ámbito de las comunidades, los cuales tienen que ser sean continuos, para constituirse en una base pedagógica-productiva, en la cual profesores y estudiantes trabajen de la mano con las comunidades de la región y del país.

Finalmente se recomienda seguir aplicando en la comunidad las estrategias identificadas en el proceso de evaluación a la hora efectuarse procesos de formación y extensión, puesto que se determinaron como apropiadas.

\section{REFERENCIAS BIBLIOGRÁFICAS}

1. Castillo E. Evaluación de los niveles de desarrollo sostenible en espacios territoriales (granjas de producción sostenible) en provincias centrales. Investigación y Pensamiento Crítico. 2 10-18. 2004.

2. Crona B.I., Parker J.N. Learning in support of governance: theories, methods, and a framework to assess how bridging organizations contribute to adaptive resource governance. Ecology and Society. 17 (1): Art. 32. 2012. 
3. Chapin F.S., Peterson G., Berkes F., Callaghan T., Angelstam P., Apps M., Beier C., Bergeron Y., Crépin A.S., Danell K., Elmqvist T., Folke C., Forbes B., Fresco N., Juday G., Niemeld J., Shvidenko A., Whitema G. Resilience and vulnerability of northern regions to social and environmental change. Ambio. 33 (6): 344-349. 2004.

4. de Zubiría J. Los modelos pedagógicos: hacia una pedagogía dialogante. Cooperativa Editorial Magisterio, Bogotá, Colombia. 249 p. 2006.

5. Delanty G. Community. Comunidad, educacion ambiental y ciudadanıa. Editorial GRAO, Societat Balear d'Educació Ambiental (SBEA) y Societat Catalana d'Educació Ambiental (SCEA), Barcelona, España. 279 p. 2006.

6. GNC, Gobierno Nacional de Colombia. Política Nacional de Seguridad Alimentaria y Nutricional. Documento Conpes Social N. 113. Ministerio de la Protección Social, Ministerio de Agricultura y Desarrollo Rural, Ministerio de Educación Nacional, Instituto Colombiano de Bienestar Familiar, Instituto Colombiano de Desarrollo rural, Consejo Nacional de Política Económica Social, Departamento Nacional de Planeación Bogotá, Colombia. 47 p. 2007.

7. IDEAM, Instituto de Hidrología, Metereología y Estudios Ambientales. Tiempo y clima. 2016. Recuperado 16 de Diciembre 2016. Disponible En: http://www.ideam.gov.co/

8. Khan N., Trivedi P. Gender differences and sustainable consumption behavior. British Journal of Marketing Studies. 3 (3): 29-35. 2015.

9. Martínez R. Sistemas de producción agrícola sostenible. Tecnología en Marcha. 22 (2): 23-39. 2009.

10. Moreno S., Fidélis T. A proposal to explore the role of sustainability indicators in local governance contexts: The case of Palmela, Portugal. Ecological Indicators. 23 608-615. 2012.

11. MSPS, FAO, Documento técnico de la situación en Seguridad Alimentaria y Nutricional. Proyecto UTF/COL/039. Ministerio de Salud y Protección Social; Organización de las Naciones Unidas para la Alimentación y la Agricultura, Bogotá, Colombia. 115 p. 2012.

12. MSSSI, Ministerio de Sanidad, Servicios Sociales e Igualdad. Impactos del cambio climático en la salud. Resumen ejecutivo. Observatorio de Salud y Cambio Climático, 27 p. 2013.

13. Ortegón E., Pacheco J.F., Prieto A. Metodología del marco lógico para la planificación, el seguimiento y la evaluación de proyectos y programas. United Nations Publications, Santiago de Chile. 124 p. 2005.

14. Pérez E., Sánchez J. La educación comunitaria: Una concepción desde la pedagogía de la esperanza de Paulo Freire. Revista Venezolana de Ciencias Sociales. 9 (2): 317-329. 2005.

15. PESA, Programa Especial para la Seguridad Alimentaria en Centroamérica. Seguridad alimentaria nutricional, conceptos básicos. FAO, Organización de la Naciones Unidas para la Alimentación y la Agricultura, 8 p. 2011.

16. Reales L.J., Arce J.A., Heredia F.A. La organización educativa y su cultura: una visión desde la postmodernidad. Laurus. 14 (26): 319-346. 2008. 